\title{
3-Dimensional Mapping and Radiofrequency Ablation of Atrial Flutter in a Patient with Interrupted Inferior Vena Cava
}

\author{
Siddharth Mukerji ${ }^{1}$, Chotikorn Khunnawat ${ }^{1}$, \\ Sricharan Kantipudi ${ }^{1}$, Atul Khasnis ${ }^{1}$, Sundar \\ Sankaran ${ }^{1}$, Ranjan K. Thakur ${ }^{1}$, and Krit \\ Jongnarangsin ${ }^{2}$ \\ ${ }^{1}$ Division of Cardiology, Michigan State University, East \\ Lansing, Michigan; ${ }^{2}$ Department of Internal Medicine, Division \\ of Cardiovascular Medicine, University of Michigan, Ann Arbor, \\ Michigan
}

\begin{abstract}
The presence of isolated interrupted inferior vena cava (IVC) is very rare. Though the occurrence of typical atrial flutter in this setting has recently been described, the use of 3-dimensional activation mapping to aid the management of such patients has not yet been described. We report the successful ablation of this arrhythmia in a 63-year-old woman using the superior route through the internal jugular vein with the help of a mapping system.
\end{abstract}

Key Words. atrial flutter, catheter ablation, inferior vena cava, azygos vein

\section{Introduction}

Interruption of the inferior vena cava (IVC) is a rare developmental anomaly. It is believed to be associated with various associated cardiac anomalies as a component of heterotaxy syndromes. The association of conduction abnormalities other than heart block has not been described in such syndromes [1]. Cavotricuspid isthmus dependent atrial flutter is a common form of macroreentrant tachycardia which can occur in a clockwise or counterclockwise direction [2]. We report the successful catheter ablation of atrial flutter in a patient with isolated interrupted IVC with the help of a 3-dimensional mapping system.

\section{Case Report}

A 63-year-old woman with a history of hypertension but no prior cardiac disease was admitted with atrial flutter with 2:1 AV conduction. The EKG was consistent with counterclockwise atrial flutter. Rate control was achieved using intra- venous diltiazem. A transesophageal echocardiography demonstrated no structural heart disease or evidence of intracardiac thrombus. An electrophysiologic (EP) study and catheter radiofrequency (RF) ablation was recommended.

During the EP study, it was difficult to advance a quadripolar catheter to the right atrium via the right femoral vein. The catheter entered the right atrium at an unusual location. Venography of the IVC was performed (Fig. 1). This demonstrated interruption of the IVC and the presence of a large azygos vein connection emptying into the superior vena cava and right atrium.

The procedure was aborted due to the unexpected abnormality. Subsequent magnetic resonance angiography and venography (MR) showed the absence of the hepatic IVC. The hepatic venous drainage from the right and left hepatic veins drained directly into the right atrium without evidence of reformation of a hepatic IVC. The contribution to the azygos veins from below the renal veins appeared to be from a left-sided IVC. MRI findings confirmed the diagnosis of interrupted IVC with azygos continuation.

The patient was brought back to the electrophysiology laboratory for a repeat electrophysiologic study. Because of the interrupted IVC, right heart catheterization was performed via left subclavian and right internal jugular venous access. The coronary sinus was cannulated by a decap-

Address for correspondence: Krit Jongnarangsin, MD, Department of Internal Medicine, Division of Cardiovascular Medicine, 1500 E. Medical Center Drive, VAMC 111 A, Box 2399, Ann Arbor, MI 48109. E-mail: kritj@med.umich.edu

Received 13 July 2005; accepted 22 August 2005 


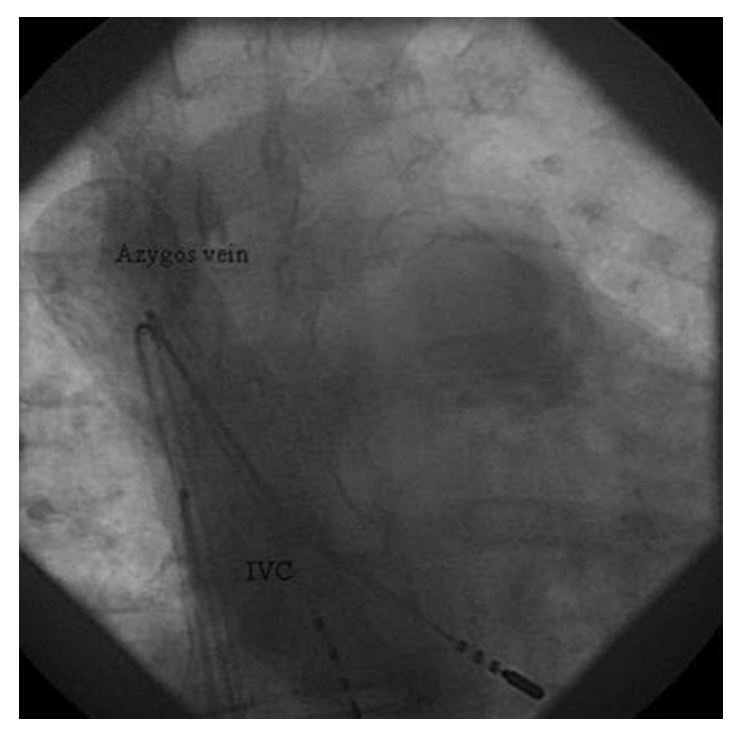

Fig. 1. Cine venogram showing interruption of the inferior vena cava (IVC) with a dilated azygos vein (AV). The catheter is seen to enter the right atrium at a higher level via the azygos vein.

olar catheter, which was inserted via left subclavian vein. A steerable catheter (Navistar ${ }^{\mathrm{TM}}$, Biosense-Webster) with an $8 \mathrm{~mm}$ tip was inserted into the right atrium via the right jugular vein for mapping and ablation. Right atrium, SVC- and hepatic veins were reconstructed using a 3-dimensional mapping system $\left(\right.$ CARTO $^{\mathrm{TM}}$, Biosense-Webster). Activation sequence mapping of atrial flutter was then performed and showed counterclockwise propagation of activation wave front around tricuspid annulus (Fig. 2). Radiofrequency ablation was performed to create a lin- ear lesion from tricuspid valve to hepatic vein, which resulted in termination of atrial flutter. Ablation was continued until a transisthmus conduction block was achieved. A repeat atrial activation sequence mapping was performed during atrial pacing from a pair of electrodes on the decapolar catheter at the coronary sinus os. Propagation map demonstrated a line of complete conduction block from the tricuspid valve to the common hepatic vein. The patient tolerated the procedure well. There were no procedure-related complications. She remained in normal sinus rhythm with no recurrence of symptoms at one year of follow-up.

\section{Discussion}

Heterotaxy (syndrome) is defined as the abnormal arrangement of visceral organs and blood vessels differing from the arrangement of situs solitus and situs inversus [1,3]. Generally, a constellation of cardiac, vascular and visceral abnormalities comprise these syndromes in which isomerism is a salient feature. Isomerism refers to symmetrical morphology of the normally asymmetrical organs. The cardiac anomalies include ventricular septal defect (VSD), double-outlet right ventricle and partial anomalies of the systemic and pulmonary veins $[1,3,4]$. However, only $5-10 \%$ of these patients have normal hearts to minor cardiac defects.

Isolated interruption of the IVC with azygos continuation below the level of hepatic veins is extremely rare. The reported prevalence is $0.6-$ $2.0 \%$ in patients with congenital heart disease. In normal patients the prevalence has been found to be less than $0.3 \%$ [5-7].

In these patients (where the IVC terminates below the hepatic veins) the dilated azygos and

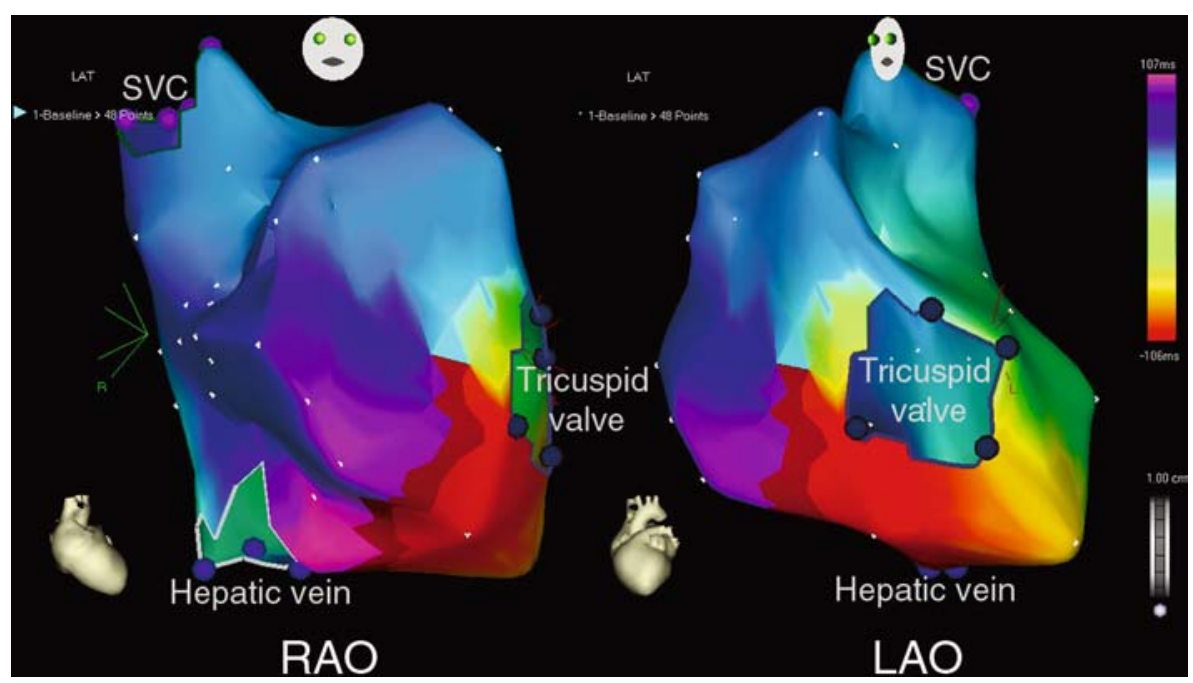

Fig. 2. Electroanatomic mapping showing the point of earliest activation adjacent to the point of the latest activation around the tricuspid annulus in a counter-clockwise direction. The hepatic vein serves as the posterior boundary. 
hemiazygos veins which eventually empties into the SVC accommodate systemic venous flow [6-8]. In our patient, the infrahepatic IVC continued as the azygos vein and the hepatic segment drained directly into the right atrium. In addition to the present case, there may be continuation to the hemiazygos vein to the SVC or anomalous intrahepatic veins $[7,9]$.

Most of the isolated anomalies are detected incidentally. The clinical implications documented thus far include presentation as a mediastinal mass or a paracardiac mass on radiography. The other manifestations include recurrent deep vein thrombosis or sick sinus syndrome and complete atrioventricular blocks. The association of counter clockwise atrial flutter with these syndromes has been reported recently [10].

It has, only in recent times, been recognized that a functional block based on anisotropic conduction was imperative in determining the atrial flutter circuit [2]. The tricuspid ring forms the anterior boundary of typical isthmus dependent atrial flutter while the posterior boundary is the orifice of the IVC, terminal crest and SVC. The circuit moves along the septal and anterior wall of the right atrium with the superior part of the right atrium on the top and the IVC-tricuspid ring isthmus below. Blocking of the isthmus completely and bidirectionally is essential for success [2,11].

On the EP study and 3-D activation mapping (Fig. 2) in our patient, it was evident that the atrial flutter was sustained using the macro reentry circuit with conduction along the interatrial septum (caudo-cranially) and the free right atrial wall (cranio-caudally) in a counterclockwise manner. The hepatic drainage orifice in the right atrium acted much like the IVC in providing the anatomical barrier posteriorly. The isthmus was difficult to reach via the femoral venous access due to the acute angle at the connection of azygos vein and SVC. We found that catheter ablation of the isthmus was easier to perform via the right internal jugular vein. Termination of atrial flutter by creating a line of conduction block at the isthmus between tricuspid annulus and the orifice of the hepatic vein confirmed the above mechanism of atrial flutter. The 3-D mapping performed earlier guided the successful ablation of this arrhythmia.

\section{Conclusion}

Typical counterclockwise isthmus dependent atrial flutter can be seen in association with in- terrupted IVC with azygos connection. The mechanism of the tachycardia is similar to typical atrial flutter commonly seen in patients with normal heart except that the orifice of the hepatic vein serves as a posterior boundary instead of the IVC. Although the isthmus is difficult to approach due to interruption of the IVC, and other routes have been described [12], we successfully performed catheter ablation via the superior approach. This was aided by the presence of a 3-dimensional activation mapping system.

\section{References}

1. Berg C, Geipel A, Smrcek J, Krapp M, Germer U, Kohl T, Gembruch U, Baschat AA. Prenatal diagnosis of cardiosplenic syndromes: A 10-year experience. Ultrasound $\mathrm{Ob}$ stet Gynecol 2003;22(5):451-459.

2. Cosio FG. Atrial flutter update. Card Electrophysiol Rev 2002;6(4):356-364.

3. Winer-Muram HT, Tonkin IL. The spectrum of heterotaxic syndromes. Radiol Clin North Am 1989;27(6):1147-1170.

4. Ho SY, Cook A, Anderson RH, Allan LD, Fagg N. Isomerism of the atrial appendages in the fetus. Pediatr Pathol 1991;11(4):589-608.

5. Timmers GJ, Falke TH, Rauwerda JA, Huijgens PC. Deep vein thrombosis as a presenting symptom of congenital interruption of the inferior vena cava. Int $J$ Clin Pract 1999;53(1):75-76.

6. Vijayvergiya R, Bhat MN, Kumar RM, Vivekanand SG, Grover A. Azygos continuation of interrupted inferior vena cava in association with sick sinus syndrome. Heart 2005;91(4):e26.

7. Chuang VP, Mena CE, Hoskins PA.Congenital anomalies of the inferior vena cava. Review of embryogenesis and presentation of a simplified classification. $\mathrm{Br} J$ Radiol 1974;47(556):206-213.

8. Balkanci F, Ozmen MN. Case report: Interruption of the inferior vena cava with anomalous intrahepatic continuation. Br J Radiol 1993;66(785):457-459.

9. Beedie RJ, Yeo W, Morcos SK. Congenital absence of the intrahepatic segment of the inferior vena cava with azygos continuation presenting as a mediastinal mass. Postgrad Med J 1989;65(762):253-255.

10. Malavasi VL, Casali E, Rossi L, Modena MG. Radiofrequency catheter ablation of common atrial flutter in a patient with anomalous inferior vena cava and azygos continuation. Pacing Clin Electrophysiol 28(7):733-735.

11. Cosio FG, Lopez-Gil M, Goicolea A, Arribas F, Barroso JL. Radiofrequency ablation of the inferior vena cava-tricuspid valve isthmus in common atrial flutter. Am $J$ Cardiol 1993;71(8):705-709.

12. Fischbach P, Campbell RM, Hulse E, Mosca R, Armstrong B, Lloyd TR, Dick M 2nd.Transhepatic access to the atrioventricular ring for delivery of radiofrequency energy. $J$ Cardiovasc Electrophysiol 1997;8(5):512-516. 